\title{
GW Calculation of a Carbon Oxide Molecule Using an All-Electron Mixed-Basis Approach
}

\author{
Soh Ishii ${ }^{1}$, Kaoru Ohno ${ }^{2}$ and Yoshiyuki Kawazoe ${ }^{1}$ \\ ${ }^{1}$ Institute for Materials Research, Tohoku University, Sendai 980-8577, Japan \\ ${ }^{2}$ Department of Physics, Graduate school of Engineering, Yokohama National University, Yokohama 240-8501, Japan
}

\begin{abstract}
An ab-initio calculation for a carbon oxide molecule using the Green's function approach within the $G W$ approximation was performed. We use an all-electron mixed-basis approach, where one wave function is expanded using both plane waves and atomic orbitals. This approach has an advantage to describe the wave function of a carbon and oxide, compared with a pseudopotential approach requiring higher cutoff energy. Obtaied $G W$ quasiparticle energies are in good agreement with avairable experimental value and previous $G W$ calculation.
\end{abstract}

(Received December 24, 2003; Accepted February 20, 2004)

Keywords: GW approximation, carbon oxide, first-principles, electron correlation, ionization potential, electron affinity

\section{Introduction}

The carbon oxide molecule is important from the viewpoint of not only engieering side but also physical side and has been widely investigated both experimentally and theoretically. In the investigation of such small molecules, ionization potentials (IPs) and electron affinities (EAs) are important because these quantities, for example, play an significant role to deteremine chemical reactions and optical properties. To evaluate IPs and EAs by means of $a b$-initio calculations, there are at least two methods. The first method is taking total energy difference between a cation (anion) and neutral molecules for evaluating the IP (EA). However, one needs calculate total energy at least three times to obtain both IP and EA. The second is using the Koopmans theorem: the absolute value of the highest occupied molecular orbital (HOMO) and the lowest unoccupied molecular orbital (LUMO) energy is the IP and EA, respectively. The approach using this theorem is significantly different from the first one. This method has an advantage from the viewpoint of the times of calculations:this method simultaneously gives all the energy levels (for example, one can get the IP and EA at the same time). In ab-initio calculations, one of the standard methods is the local density approximation (LDA) ${ }^{1)}$ within the density functional theory (DFT), ${ }^{2)}$ which is a very good theory for the description of the ground state properties. However, ionization potentials (electron affinities) of the LDA via Koopmans theorem underestimates (overestimates) experimental IPs (EAs) very much. Note that the absolute value of the HOMO energy is identical to IP even within the density functional theory. ${ }^{3}$

In the present study, we take the latter approach and employ the Green's function approach within the $G W$ approximation, ${ }^{4}$ which gives the one-particle excitation energy correctly. In the $G W$ approximation, electron selfenergy operator is defined by using one-particle Green's function and dynamically screened Coulomb interaction. Historically, ab-initio $G W$ calculations were firstly performed by Hybertsen and Louie $^{5)}$ for typical semiconductors such as silicon and germanium successfully. However, the number of papers of the $G W$ calculations for isolated systems such as molecules and clusters are small. ${ }^{6-12)}$ In addition, almost all calculations employ a pseudopotential approach. We successfully applied ab-initio $G W$ calculations to small alkali-metal clusters ${ }^{9,10)}$ and silicon clusters, ${ }^{11)}$ using an allelectron mixed-basis approach.

In the present paper, we perform an ab-initio $G W$ calculation for a carbon oxide molecule using an all-electron mixed-basis approach and compare with other calculatioins $^{12)}$ and experimental data. In the next section, we explain our method briefly. Sec. 3 is devoted to the results and discussion. Sec. 4 is summary.

\section{Methodology}

We employ an all-electron mixed-basis approach, where a wave function is expanded using both plane waves and atomic orbitals, where the one-particle wave function is represented by plane waves (PWs) and atomic orbitals (AOs) to take into account both the core electron states and the empty free-electron-like states accurately. This approach has been successfully applied not only to isolated systems but also to infinite systems. ${ }^{9,13,14)}$ This approach also has an advantage for the study of second row elements such as carbon, nitrogen, oxygen, and so on because the cut-off energy for the PWs needed in the present approach is much smaller than that of a pseudopotential approach (see below). As an atomic orbital, we employ Herman-Skillman code ${ }^{15)}$ to make atomic orbitals.

In the $G W$ approximation (GWA), the one-electron selfenergy operator $\Sigma\left(\mathbf{r}, \mathbf{r}^{\prime} ; \omega\right)$ is given by (apart from the Hartree potential) $)^{4)}$

$$
\Sigma\left(\mathbf{r}, \mathbf{r}^{\prime} ; \omega\right)=\frac{i}{2 \pi} \int G\left(\mathbf{r}, \mathbf{r}^{\prime} ; \omega+\omega^{\prime}\right) W\left(\mathbf{r}, \mathbf{r}^{\prime} ; \omega^{\prime}\right) e^{i \eta \omega^{\prime}} d \omega^{\prime},
$$

where $G$ and $W$ denote the one-particle Green's function and the dynamically screened Coulomb interaction,respectively. $\eta$ is a positive infinitesimal number. $W$ is usually evaluated within the random phase approximation. The Fock exchange part of the self-energy, $\Sigma_{\mathrm{x}}$, is obtained by replacing $W$ with the bare Coulomb interaction in eq. (1), while we call $\Sigma_{\mathrm{c}}=$ $\Sigma-\Sigma_{\mathrm{x}}$ the correlation part. Note that self-energy operator is 
non-local in space and frequency dependent.

In the present study, we use the LDA wave functions and eigenvalues to evaluate $G$ and $W$ from the viewpoint of the perturbation theory. The $G W$ quasiparticle energy is then given by

$$
\begin{aligned}
E_{i}^{\mathrm{GWA}} & =E_{i}^{\mathrm{LDA}}+z\left\langle i\left|\Sigma\left(E_{i}^{\mathrm{LDA}}\right)-\mu_{\mathrm{xc}}^{\mathrm{LDA}}\right| i\right\rangle, \\
z & =\left[1-(\partial \Sigma(\omega) / \partial \omega)_{E_{i}^{\mathrm{LDA}}}\right]^{-1},
\end{aligned}
$$

where $E_{i}^{\mathrm{LDA}}$ and $\mu_{\mathrm{xc}}^{\mathrm{LDA}}$ are the LDA eigenvalue and exchange-correlation potential, respectively. Equation (2) has been widely used in the literature and is known to give good quasiparticle energies for moderately correlated electron systems ${ }^{13)}$ (see sec. 3).

The bond length is taken to be $0.116 \mathrm{~nm}$, referred to the experimental value. ${ }^{16)}$ We use an fcc supercell with a cubic edge of $0.75 \mathrm{~nm}$. This is large enough to make interactions between the molecules negligible. We introduce the spherical cut-off of the Coulomb potential. ${ }^{6,9)}$ In the calculation of the LDA, the cut-off energy for the PWs is taken to be $38 \mathrm{Ry}$, smaller than the value of the pseudopotential approach, $70 \mathrm{Ry}^{12)}$ (see above). In the evaluation of $\Sigma_{\mathrm{c}}$, the cut-off energy is taken to be $28 \mathrm{Ry}$. We adopt the generalized plasmon-pole (GPP) model ${ }^{5)}$ and use 600 empty levels, corresponding to $90 \mathrm{eV}$ in the calculations. The GPP model reproduces the experimental quasiparticle energies well. ${ }^{5,10,11)}$ The core contribution is ignored in $\Sigma_{\mathrm{c}}$. On the other hand, for the evaluation of $\Sigma_{\mathrm{x}}$ in the Fourier space, we use the cut-off energy of 100 Ry to take into account the core contribution. The core contribution to $\Sigma_{\mathrm{x}}$ is very important and is fully considered in the calculations. We carefully checked that all contributions are well converged with these cut-off energies and the number of empty levels within an accuracy of $0.1 \mathrm{eV}$. Other technical details of the present calculations are explained in Ref. 9).

\section{Results and Discussion}

In Table 1 , we show the $G W$ quasiparticle energies, $E_{i}^{\mathrm{GWA}}$, for the HOMO (highest occupied molecular orbital) and the LUMO (lowest unoccupied molecular orbital) levels, as well as the experimental $\mathrm{IP}^{16)}$ with minus signs, $E_{i}^{\mathrm{EXP}}$. Table 1 lists also different contributions to $E_{i}^{\mathrm{GWA}}$ in eq. (1). To our knowledge, there is no experimental data for EA. Note once again that the absolute values of both the quasiparticle energies and DFT eigenvalues for the HOMO ( $\sigma$ character) and LUMO ( $\pi$ character) levels represent, respectively, the

Table 1 Contributions to the GWA quasiparticle energies (in $\mathrm{eV}$ ) for the HOMO and LUMO level of a carbon oxide molecule and the experimental ionization potential ${ }^{16)}$ with minus signs $\left(E_{i}^{\mathrm{EXP}}\right) . \quad \mu_{\mathrm{xc}, i}^{\mathrm{LDA}}=\left\langle i\left|\mu_{\mathrm{xc}}^{\mathrm{LDA}}\right| i\right\rangle$, $\Sigma_{\mathrm{x}, i}=\left\langle i\left|\Sigma_{\mathrm{x}}\right| i\right\rangle$ and $\Sigma_{\mathrm{c}, i}\left(E_{i}^{\mathrm{LDA}}\right)=\left\langle i\left|\Sigma_{\mathrm{c}}\left(E_{i}^{\mathrm{LDA}}\right)\right| i\right\rangle$ are the expectation values of the LDA exchange-correlation potential, exchange part, and the correlation part of the self-energy $\Sigma$, respectively. The final result $E_{i}^{\mathrm{GWA}}$ is evaluated using eq. (2)

\begin{tabular}{llllllll}
\hline & & $E_{i}^{\mathrm{LDA}}$ & $\mu_{\mathrm{xc}, i}^{\mathrm{LDA}}$ & $\Sigma_{\mathrm{x}, i}$ & $\Sigma_{\mathrm{c}, i}\left(E_{i}^{\mathrm{LDA}}\right)$ & $E_{i}^{\mathrm{GWA}}$ & $E_{i}^{\mathrm{EXP}}$ \\
\hline CO & HOMO & -9.21 & -14.91 & -20.35 & +0.15 & -13.96 & $-14.01^{\mathrm{a}}$ \\
& HOMO & & & & & $-14.1^{\mathrm{b}}$ & $-14.01^{\mathrm{a}}$ \\
& LUMO & -2.43 & -14.21 & -7.92 & -1.78 & +1.77 & - \\
\hline
\end{tabular}

${ }^{a}$ Ref. 16)

${ }^{\mathrm{b}}$ Ref. 12)
IP and the EA. ${ }^{3)}$ From the Table 1, although the LDA eigenvalue of the HOMO level underestimates the experimental IP by about $5 \mathrm{eV}$, the $G W$ quasiparticle energy is in good agreement with the experimental value ${ }^{16)}$ and the previous $G W$ result.

$\Sigma_{\mathrm{x}}$ plays an important role to reproduce the experimental IP. $\Sigma_{\mathrm{x}}$ is Fock-exchange energy evaluated using the LDA wave functions, comparable to the Hartree-Fock (HF) exchange energy using the HF wave functions. The HF exchange energy is, from another point of view, a first order term in terms of the expansion of the bare Coulomb interaction. On the other hand, $\Sigma_{\mathrm{c}}$ is the one of the second order term in terms of the bare Coulomb interaction. Because of that, the absolute value of $\Sigma_{\mathrm{x}}$ is larger than that of $\Sigma_{\mathrm{c}}$.

The LUMO energy obtained by the present $G W$ calculation is positive value, although the LDA eigenvalue is negative. Let us discuss this. The present $G W$ calculation employs eq. (2), based on the assumption that the LDA wave functions are very close to the exact wave functions. This is true for the wave functions under the bottom of continuum level $(E<0)$. Above the continuum level $(E>0)$, however, the LDA wave fucntions are not good approximation because one cannot ignore the interactions with plane wave solutions (continuum state). In fact, it was pointed out that the difference between the LDA wave functions and $G W$ wave functions is large at $E>0 .^{7,9)}$ To take into account this effect, one must calculate the off-diagonal elements of $\Sigma-\mu_{\mathrm{xc}}^{\mathrm{LDA}}$, $\left\langle i\left|\Sigma-\mu_{\mathrm{xc}}^{\mathrm{LDA}}\right| j\right\rangle$, then diagonalize this matrix. This effect has a tendency to make energy levels above the bottom of the continuum level decrease. ${ }^{7,9)}$ However, to perform this calculatinon is computationally very demanding even if one uses a supercomputer. The absolute value of the LUMO energy obtained by the present $G W$ calculation is not reliable because of the above reason. However, at least one can mention that a carbon oxide molecule cannot attract an extra electron.

\section{Summary}

We performed the $G W$ calculation for a carbon oxide molecule using the all-electron mixed-basis approach, where a wave fucntion is expanded using the plane waves and atomic orbitals. Although the absolute value of the LDA eingenvalue of the highest occupied energy level is smaller than the experimental ionization potential by about $5 \mathrm{eV}$, the $G W$ quasiparticle energy is in very good agreement with experimental value. The present result is also in good agreement with previous calculation. The lowest unoccupied energy level of the present $G W$ calculation is positive. That is, a carbon oxide molcule cannot attract an extra electron.

\section{Acknowledgments}

The authors thank the Center for Computational Materials Science of the Institute for Materials Research, Tohoku University for the support of the SR8000 supercomputing facilities. One of the authors (S.I.) thanks to Special Coordination Funds for Promoting Science and Technology from the Ministry of Education, Culture, Sports, Science, and Technology of the Japanese government. 


\section{REFERENCES}

1) W. Kohn and L. J. Sham: Phys. Rev. 140 (1965) A1133-A1138.

2) P. Hohenberg and W. Kohn: Phys. Rev. 136 (1964) B864-B871.

3) C.-O. Almbladh and U. von Barth: Phys. Rev. B 31 (1985) 3231-3244.

4) L. Hedin: Phys. Rev. 139 (1965) A796-A823.

5) M. S. Hybertsen and S. G. Louie: Phys. Rev. Lett. 55 (1985) 1418 1421.

6) G. Onida, L. Reining, R. W. Godby, R. D. Sole and W. Andreoni: Phys. Rev. Lett. 75 (1995) 818-821.

7) J. C. Grossman, M. Rohlfing, L. Mitas, S. G. Louie and M. L. Cohen: Phys. Rev. Lett. 86 (2001) 472-475.

8) M. Rohlfing and S. G. Louie: Phys. Rev. Lett. 80 (1998) 3320-3323.

9) S. Ishii, K. Ohno, Y. Kawazoe and S. G. Louie: Phys. Rev. B 63 (2001) 155104-1-155104-6.
10) S. Ishii, K. Ohno, Y. Kawazoe and S. G. Louie: Phys. Rev. B 65 (2002) 245109-1-245109-6.

11) S. Ishii, K. Ohno, V. Kumar and Y. Kawazoe: Phys. Rev. B 68 (2003) 195412-1-195412-5.

12) S. Ismail-Beigi and S. G. Louie: Phys. Rev. Lett. 90 (2003) 076401-1076401-4.

13) See, for example, K. Ohno, K. Esfarjani and Y. Kawazoe: Computational Materials Science, Solid-State Sciences, Vol. 129 (SpringerVerlag, Berlin, Heidelberg, 1999); and references therein.

14) S. Ishii, K. Ohno and Y. Kawazoe: Mater. Trans., JIM 40 (1999) 12091212.

15) F. Herman and S. Skillman: Atomic Structure Calculations, (PrenticeHall, Englewood Cliffs, NJ, 1963).

16) NIST Chemistry WebBook, NIST Standard Rerefence Database, ed. by P. J. Linstrom and W. G. Mallard, 2001 (http://webbook.nist.gov). 